\title{
Optimized Production of Biodiesel Using Internet of Things Sensed Temperature with Hybrid Particle Swarm Optimization
}

\author{
G. Loganathan $\mathbb{D}^{1}$ and M. Kannan ${ }^{2}$ \\ ${ }^{1}$ Department of Mechanical Engineering, PERI Institute of Technology, Mannivakkam, Chennai-600 048, Tamilnadu, India \\ ${ }^{2}$ Department of Mechanical Engineering, KCG College of Technology, Chennai-600097, Tamilnadu, India
}

Correspondence should be addressed to G. Loganathan; logumech232@gmail.com

Received 6 August 2021; Revised 1 December 2021; Accepted 20 December 2021; Published 10 January 2022

Academic Editor: Susana Casal

Copyright (c) 2022 G. Loganathan and M. Kannan. This is an open access article distributed under the Creative Commons Attribution License, which permits unrestricted use, distribution, and reproduction in any medium, provided the original work is properly cited.

\begin{abstract}
Biofuel production offers a non-fossil fuel that can be utilized in modern engines without any redesign. Regardless of receiving rising attention, many researchers have explored microalgae-based biofuel production and found biodiesel production is costeffective compared to petroleum-centered conventional fuels. The primary reason is that the lipid accumulation of microalgae is possible. An efficient technique is proposed for optimized biodiesel manufacturing with microalgae through an IoT device with the hybrid particle swarm optimization (HPSO) algorithm for elapsing such drawbacks. First, the component of biodiesel is determined. Then, from the components, the temperature value is sensed through the IoT device. Based on the obtained temperature, the reaction parameters are optimized with HPSO to increase productivity and reduce cost. Finally, we observed performance and comparative analysis. The experimental results contrasted with the existent particle swarm optimization (PSO) and genetic algorithm (GA) concerning iteration's temperature, concentration, production, and fitness. The present HPSO algorithm has differed from the existing PSO and GA concerning IoT sensed temperature and production function. Fitness value and instance concentration are the performance parameters. It varies based on the iteration values. Thus, the proposed optimized biodiesel production is advanced when weighed down with the top-notch methods.
\end{abstract}

\section{Introduction}

The rapid increase in the oil price, the requirement for energy, concerns about its availability, and climate change led to the search for renewable energy to keep to the demands investigated by Valizadeh et al. [1]. Yu et al. [2] and Sanli et al. [3] analyzed and reported that the biodiesel is stated as FA-alkyl monoesters emanated as renewable feedstocks, like vegetable oils and animal fats. Globally, biodiesel is chiefly produced by the transesterification (TE) process wherein oil (edible or nonedible) and alcohol (ethanol, methanol, propanol) reacted in the presence of a catalyst (homogeneous, heterogeneous, along with enzymatic). Advantages of biodiesel such as complete biodegradability, lack of sulfur particles, and particulate emissions, nontoxicity, and a fall in carbon monoxide along with carbon dioxide emissions are reported in $[4,5]$.
Vegetable oil fuels are becoming more attractive because of their renewability, energy security, and higher energy content close to the petroleum-centered powers. The biodiesel's properties resemble those of the standard diesel fuel; in addition, it can well be employed as a fuel mix or as a substitution for diesel fuel. Nowadays, disparate vegetable oils, say, soybean, camelina, sunflower, rapeseed, and palm oils, are being utilized for biodiesel production [6-9].

The transesterification is characteristically carried out utilizing primary and secondary alcohols [10]. Numerous parameters affect the biodiesel yield by transesterifying vegetable oils: reaction temperature, alcohol/oil molar ratio, agitation speed, catalyst concentration, and reaction time. Transesterification optimization needs loads of experiments and mathematical equipment to envisage every process parameter's impact. The response surface method 
was successfully utilized for the optimization of biodiesel synthesis using various raw materials and catalysts [11].

Biodiesel production expenses are highly reliant on raw materials; additionally, the price of vegetable oils/animal fats is typically a primary contributor to the cost, at about $70 \%$. Biodiesel produced as virgin vegetable oils costs more than petro-diesel. It is a chief obstacle to biodiesel commercialization. Consequently, it is essential to recognize cheaper raw oils to lessen the production expenditure of biodiesel. From this viewpoint, the utilization of WCO and nonedible fats, such as Jatropha curcas, catfish, rubber, and tobacco oils, is an apt option for lessening the expenditure of raw materials [12]. Likewise, the other raw materials, explicitly long-chain alcohols, isopropanol, ethanol, and tert-butanol, have high miscibility with oil and low reaction time compared to methanol. However, these alcohols make the separation process arduous, requiring a higher price for separation [13]. Therefore, modelling and planning the biofuel supply chain is imperative for deciding on the whole chain's performance. Supply chain intricacy has directed to look for new optimization methods. Recently, heuristic optimization has been utilized instead of traditional techniques because of its capability to find an almost optimal solution [14].

Moreover, environmental IoT sensors have become valuable for modelling and monitoring ecological occurrences. The IoT has attracted interest in connecting everyday objects with sensors and generating a network for sending and receiving data at a lower price. Concerning the parameters of the biodiesel in production workshops that required monitoring, combined with the intricate industrial production surroundings, sensible information acquisition software and hardware platforms are constructed to realize the real-time dynamic monitoring of temperature and pressure [15]. The following contributions are provided to improve the cost-effectiveness in biodiesel production with microalgae:

(1) The novel production function for biodiesel from microalgae is proposed with concentration parameters of constituents

(2) The temperature of the biodiesel is monitored through the IoT temperature sensor

(3) The temperature obtained from the IoT is optimized through the hybrid genetic and particle swarm algorithms

(4) The performance of the optimization algorithm is compared with the basic GA and PSO

\section{Related Work}

Antony et al. [16] presented that the raw oil as 3 nonedible feedstocks, Jatropha curcas, Calophyllum Inophyllum, and Pongamia Pinnata, were mixed in equivalent proportion, and the process of biodiesel optimized. As the acidic value of the mixed feedstock was higher at two-stage, biodiesel was manufactured with esterification-transesterification. Multiple variant empirical models were utilized for optimizing the parameters; furthermore, it was discovered that the methanol concentration was the most critical parameter, followed by the catalyst concentration. About $98 \%$ biodiesel yields were attained at the optimized condition. The optimized process setting intended for the transesterification process was oil/methanol ratio $(2.5 v / v)$, duration $(95 \mathrm{~min})$, and catalyst concentration $(1.17 \% w / v)$, along with temperature $\left(53^{\circ} \mathrm{C}\right)$. The parameters are authenticated via experiments, and the biodiesel properties were tested for their accord with the ASTM standard to utilize them in the existent diesel engine.

As crude oil, the Taguchi method is used in production. Biodiesel production from crude tall oil (CTO) in the presence of the mercantile lipase catalyst optimized utilizing the Taguchi technique was exposed by Peter et al. [17]. Process parameters counting molar ratio (MR) of CTO to methanol, reaction temperature, and reaction time, along with enzyme dose were inspected for their impact upon the in situ esterification of fatty acids (FAs) on CTO to FAmethyl ester (FAME). Fractional distillation was performed for separating the FAME (biodiesel) from the remaining CTO constituent. Using the Taguchi (L9) orthogonal design, the optimal biodiesel yield was statistically envisaged and experimentally authenticated as $97.02 \%$ and also $96.57 \%$, respectively. The optimum transmutes of CTO to crude biodiesel were attained at a reaction time of sixteen $(h)$, temperature of $40^{\circ} \mathrm{C}$, and $\mathrm{MR}$ of $1: 1.5$, along with enzyme dose. The reaction time and the MR were the two most contributing aspects for the optimal transmutation.

Muhammad et al. [18] examined an optimization model of biodiesel manufacturing utilizing rapeseed oil as the feedstock along with $\mathrm{NaOCH} 3$ as a catalyst. Optimization of disparate reaction parameters, reaction time and temperature, catalyst concentration, and alcohol-to-oil MR was performed utilizing the response surface methodology (RSM). The optimum experimental stipulations aimed at biodiesel production were as follows: catalyst concentration (percentage), 0.30; $\mathrm{MR}, 6.75$; reaction temperature, $55^{\circ} \mathrm{C}$; and reaction time, sixty minutes. Under these optimum conditions, 97.5 percent yield of biodiesel was attained. The noted and envisaged values of rapeseed oil methyl esters (ROMEs) displayed a linear relationship. The fuel properties such as specific gravity $(0.876 \pm 0.01)$, flash point (168 \pm ), fire point $(197.8 \pm)$, pour point $(-7 \pm)$, cloud point $(-2 \pm)$, kinematic viscosity $\left(4.42 \pm 0.26 \mathrm{~mm}^{2} / \mathrm{sec}\right)$, and sulfur content $(0.002 \mathrm{mg} / \mathrm{Kg})$ of the generated biodiesel showed the aptness of rapeseed oil (biodiesel) as fuel. Furthermore, analyze the engine performance experiment of ROME. The outcomes exhibited that the $\mathrm{CO}$ and also particulate matter emissions were under the diesel fuel. NOx emissions were low aimed at B5, B20, B40, and B50, whereas they were high for B80 as well as B100. These outcomes showed the environmental benefits of biodiesel.

Sunil et al. [19] projected a polymath for the forecast of optimized transesterification process variables of polanga biodiesel. The developed ANN was a multiple-layer feedforward backward propagation network (5-10-1) with 5 inputs, and 10 hidden layers and 1 output layer. The input features were the MR of ethanol to oil, reaction time and 
temperature, the catalyst concentration, the agitation speed, and the output parameter. The experiment data utilized in the developed ANN were attained using RSM centered on a central composite design (CCD). The trained ANN was experimented utilizing disparate training functions of the MATLAB to envisage the best correlation coefficient of training, testing, and validation. The data produced via trained ANN were utilized by the GA concerning the best retort for disparate mixtures of variables $\left(X_{1}, X_{2}, X_{3}, X_{4}\right.$, and $\left.X_{5}\right)$ for achieving optimization. The average biodiesel yield of ninety-two percentage (by performing experiments on optimized conditions) by weight was formed in opposition to the projected value of 91.08 (\%) by weight.

Sung and Young [20] evaluated a 2-phase process on a sole reactor, which includes the re-esterification of the FFAs accompanied by crude glycerol, utilizing a tin chloride catalyst; additionally, succeeding transesterification with methanol, utilizing an alkaline catalyst, was embraced. Furthermore, every single step was optimized. This study exposed that the FFA after re-esterification needed to be about $1.5 \%$, not merely for saving glycerol and the catalyst concerned in the re-esterification, but to attain a higher biodiesel conversion amid the transesterification. The alkali catalyst was productively utilized to make biodiesel on the secondary step, and also a 92.8 percentage conversion to biodiesel was attained under the optimized stipulations (0.6 percentage catalysts in connection with WCO, $0.2 \mathrm{~mL}$ methanol/WCO, $70^{\circ} \mathrm{C}, 3 \mathrm{~h}$ ). Overall, this 2 -step process attained highly ameliorated biodiesel conversion (4.0-92.8\%) with considerably lessened reaction time (twelve to four hours) along with methanol necessities (15-0.2 mL/g-WCO).

Mubarak et al. [21] obtained an optimization of lipid extortion as of Salvinia molesta aimed at biodiesel production utilizing RSM along with its FAME scrutiny. The elevated areal productivity together with the lipid part of microalgae and aquatic weed made them the finest optional feedstocks for biodiesel production. Therefore, a proficient and economic technique of extorting lipid/oil from aquatic weed, Salvinia molesta, was a significant step towards biodiesel production. As Salvinia molesta was an unexplored feedstock, its aggregated lipid part was first measured as sixteen (\%) utilizing the Bligh and Dyer technique that was quite enough for an additional investigation. For extorting more quantity of lipid from Salvinia molesta, methanolchloroform in the ratio of $2: 1 \mathrm{v} / \mathrm{v}$ was classified as the utmost appropriate solvent system utilizing the Soxhlet apparatus. Centered upon the literature along with the initial experimentations, parameters, say, temperature, the solventto-biomass ratio, and also time, were recognized as very imperative for lipid extortion. These were afterward optimized utilizing RSM with CCD, in which experiments were executed utilizing 20 combos of these extortion parameters with Minitab 17.

Zongguo et al. [22] developed an IoT-centered system and implemented it in Suzhou, China. It enclosed the generation, transportation, collection, and final disposal of restaurant food waste (RFW) and its study on the system's software and hardware components. The results showed that the system had a strong impact. Positive outcomes included the following:

(1) Enhanced management of RFW

(2) Better law enforcement in reaction to RFW misconduct

(3) An overall decrease in illegal RFW actions and enhanced process optimization athwart the RFW value chain

Based on the HPSO-MGA algorithm, Ramasamy and Pillai [24] offer a unique dynamic resource allocation strategy. The suggested method is divided into two parts, the first of which involves feature extraction and feature reduction. The HPSO-MGA technique is then used to dynamically assign resources in the second phase. When comparing the proposed HPSO-MGA to the existing IDSA and krill herd LB in terms of average waiting time, response time, load balancing, relative error, consumption time, and throughput, the comparison result showed that the proposed HPSO-MGA took less time to allocate resources.

The literature survey observed that different materials generate biodiesel at different proportions to achieve maximum efficiency. Microalgae usage for biodiesel production is practical as it contains a large volume of carbon dioxide. In general, the lipid extraction from the microalgae is significantly less, and hence, there is a need to optimize the biodiesel production from it at a lower cost.

\section{Proposed Methodology}

Production of biodiesel is the optimization of the process because of its environmentally valuable traits and its renewable nature. The proposed work accentuates the optimized biodiesel production. The steps that are concerned in the execution of the proposed work are listed as follows:

(1) The constituent's concentration is determined.

(2) A differential model can represent the primary concentration constituents. This model is a compilation of equations that display many components' concentration present amid the biodiesel production.

(3) The cost function is determined for each constituent of the biodiesel.

(4) To augment the fuel production amid the chemical reactions, and interaction between several substances is considered.

Afterward, the optimization of the reaction parameters is performed to expand the productivity.

The production expenditure of biodiesel is optimized. It is performed utilizing the HPSO here; the temperature value is generated from the IoT device. The IoT temperature sensor monitors the esterification kettle with a temperature range of 0-200 degree Celsius. Similarly, the other two sensors are placed at the dry pots and distil towers with a temperature range of $0-100$ and $0-300$ degrees Celsius, respectively. The 
temperatures sensed from the respective components are passed onto the controller unit. Zigbee obtains the data and transmits it to the server that runs the optimization algorithm through the wireless network 802.15.4. From the obtained data, the optimization of temperature is carried out for enhanced biodiesel production. Finally, performance parameters are ascertained and executed with scrutiny. The detailed elucidation of the proposed technique is displayed in Figure 1.

3.1. Determination of the Constituents of Biodiesel. Biodiesel can well be produced from new or utilized vegetable oils and animal fat. The fuel is biologically degradable, and when it is utilized as an ingredient, it requires minimal alterations to the engine to replace gasoline. Vegetable oils are competent to generate chemical compounds, called Esther, combined with alcohol (typically methanol). These are utilized for fuel (biodiesel). Glycerol (used in pharmaceutical and cosmetic products) is made from the byproduct of the biodiesel process called transesterification. Triglycerides are the blend of an alcohol termed glycerol and also three fatty acids. TG has a more potential energetic form of sugar.

Furthermore, this molecule is chemically inert. Triglycerides are actual parts of a significantly lower density lipoprotein (VLDL) along with chylomicron (CHY). Glyceride in oilseeds, along with animal fats, is plentiful. It is broken into monoglyceride, diglyceride, and free fatty acids through natural enzymes (lipase). Glycerin has three functional hydroxyl groups: monoglyceride, diglyceride, and triglyceride. Glycerin is nothing but an alcohol 3-factor formula $\left(\mathrm{C}_{2} \mathrm{H}_{5}(\mathrm{OH})\right)$ and is a colourless, transparent liquid and sweet, odourless, and hot. In addition, it gradually absorbs the moisture in the air. It is soluble in water and ethanol but not in organic solvents, say, ether, chloroform, and benzene. Most compounds that dissolve in water/alcohol are also soluble in glycerin. In Figure 2, the production reaction of biodiesel is elucidated.

3.2. Initial Concentration Parameters. Initially, take six concentration parameters: triglycerides, diglycerides, monoglycerides, methyl ester, methanol, and glycerol. These six parameters are expressed in the following equation:

$$
\begin{aligned}
\frac{\mathrm{d} C_{T G}}{\mathrm{~d} t} & =-K_{1} C_{T G} C_{A}+K_{2} C_{D G} C_{E}, \\
\frac{\mathrm{d} C_{D G}}{\mathrm{~d} t} & =K_{1} C_{T G} C_{A}+K_{2} C_{D G} C_{E}-K_{3} C_{D G} C_{A}+K_{4} C_{M G} C_{E}, \\
\frac{\mathrm{d} C_{M G}}{\mathrm{~d} t} & =K_{3} C_{D G} C_{A}-K_{4} C_{M G} C_{E}-K_{5} C_{M G} C_{A}+K_{6} C_{G L} C_{E}, \\
\frac{\mathrm{d} C_{E}}{\mathrm{~d} t} & =K_{1} C_{T G} C_{A}-K_{2} C_{D G} C_{E}+K_{3} C_{D G} C_{A}-K_{4} C_{M G} C_{E}+K_{5} C_{M G} C_{A}-K_{6} C_{G L} C_{E}, \\
\frac{\mathrm{d} C}{\mathrm{~d} t} & =-\frac{\mathrm{d} C_{E}}{\mathrm{~d} t}, \\
\frac{\mathrm{d} C_{G L}}{\mathrm{~d} t} & =K_{5} C_{M G} C_{A}-K_{6} C_{G L} C_{E},
\end{aligned}
$$

where $C_{T G}, C_{D G}, C_{M G}, C_{A}, C_{E}$, and $C_{G L}$ are the concentrations of triglycerides, diglycerides, monoglycerides, methanol, methyl ester, and glycerol, respectively. Then, these are optimized, which is discussed next.

3.3. Optimization Using Hybrid PSO. PSO was planned on account of the social activities of the birds on a flock. In PSO, every particle fly in the search space has a velocity attuned via its flying memory along with its companion's flying experience. Moreover, every particle encompasses its objective function value ascertained by a fitness function (FF). PSO is an evolutionary algorithm that is significantly like the GA, where a populace of arbitrary solutions initializes a particular system. Here, the operations like crossover and mutation are incorporated in the GA for enhancing the optimization.

In HPSO, together with each solution, the arbitrary velocity is also assigned, forming a particle. Each particle checks its coordinates on the problem space (PS) alongside the better solution. For the remaining process to be carried out, the fitness value (FV) remains the primary consideration alluded to as pbest. Gbest in HPSO refers to the site of the predefined solution.

As a result, to tackle the biodiesel problem, this work provides an enhanced hybrid particle swarm optimization approach. This algorithm replaces the particle position and velocity update formula of the particle swarm optimization algorithm with a genetic algorithm's crossover and mutation operations. Particle swarm optimization method (PSO) and 


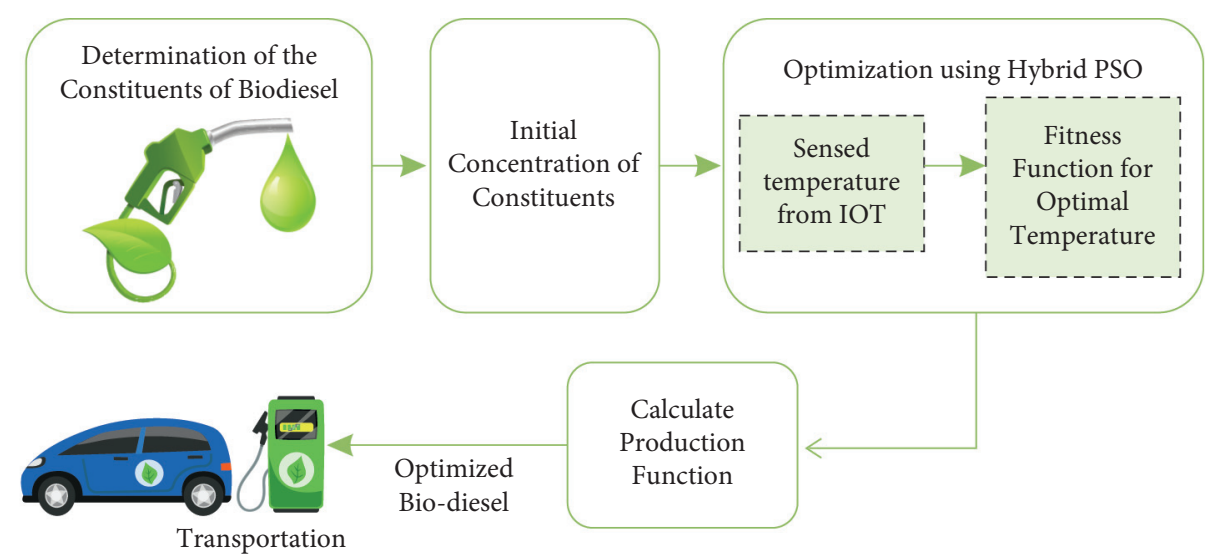

Figure 1: Block diagram for the proposed work.

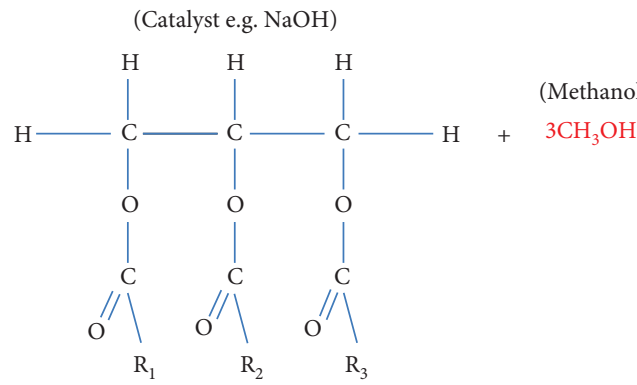

$$
\begin{aligned}
\mathrm{TG}+\mathrm{CH}_{3} \mathrm{OH} & \stackrel{\mathrm{K} 1, \mathrm{~K} 2}{\longleftrightarrow} \mathrm{DG}+\mathrm{R}_{1} \mathrm{COOCH}_{3} \\
\mathrm{DG}+\mathrm{CH}_{3} \mathrm{OH} & \stackrel{\mathrm{K} 3, \mathrm{~K} 4}{\longleftrightarrow} \mathrm{MG}+\mathrm{R}_{1} \mathrm{COOCH}_{3} \\
\mathrm{MG}+\mathrm{CH}_{3} \mathrm{OH} & \stackrel{\mathrm{K} 5, \mathrm{~K} 6}{\longleftrightarrow} \mathrm{GL}+\mathrm{R}_{1} \mathrm{COOCH}_{3}
\end{aligned}
$$<smiles>C#CC#N</smiles>

Overall reaction:

$\mathrm{TG}+\mathrm{CH}_{3} \mathrm{OH} \Leftrightarrow 3 \mathrm{RCOOCH}_{3}+\mathrm{GL}$
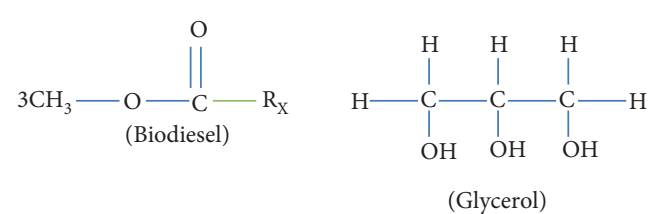

Figure 2: Biodiesel production reaction.

genetic algorithm are combined in this algorithm (GA). The HPSO thus offers a better solution. The steps are given in the following section. The general steps concerned in the HPSO with GA are exhibited in Figure 3.

3.3.1. Steps in HPSO with GA. The disparate steps concerned for applying the HPSO are delineated as follows:

(1) Foremost, initialize a populace of particles (solutions) with position and also velocity selected arbitrarily for $n$-variable in the PS.

(2) For each arbitrarily generated particle, estimate the optimized fitness function on $\mathrm{n}$-variables. In this proposed technique, the fitness function is gauged using the following equation:

$$
k_{i}=a_{i} e^{-\left(-b_{i} / T\right)},
$$

where $A=[0 ; 0 ; 0 ; 1 ; 0 ; 0]$ in which 0 and 1 values are assigned for the $a_{i}$ and $b_{i}$, respectively. $T$ (Temperature) is the control value. The temperature values are generated from the IoT device. In a particular period of time, the IoT device sends the temperature value.

Here, the best fitness function values are summarized using the following expression:

$$
\begin{aligned}
\frac{\mathrm{d} C_{E}}{\mathrm{~d} t}= & K_{1} C_{T G} C_{A}-K_{2} C_{D G} C_{E}+K_{3} C_{D G} C_{A}-K_{4} C_{M G} C_{E} \\
& +K_{5} C_{M G} C_{A}-K_{6} C_{G L} C_{E} .
\end{aligned}
$$

Now, compare this fitness value with the particles' pbest value. If this current fitness value is superior compared to the pbest, then choose the current fitness value as the pbest for further processing:

(1) This fitness value is contrasted to the best initial values. If the current value is better, update the 


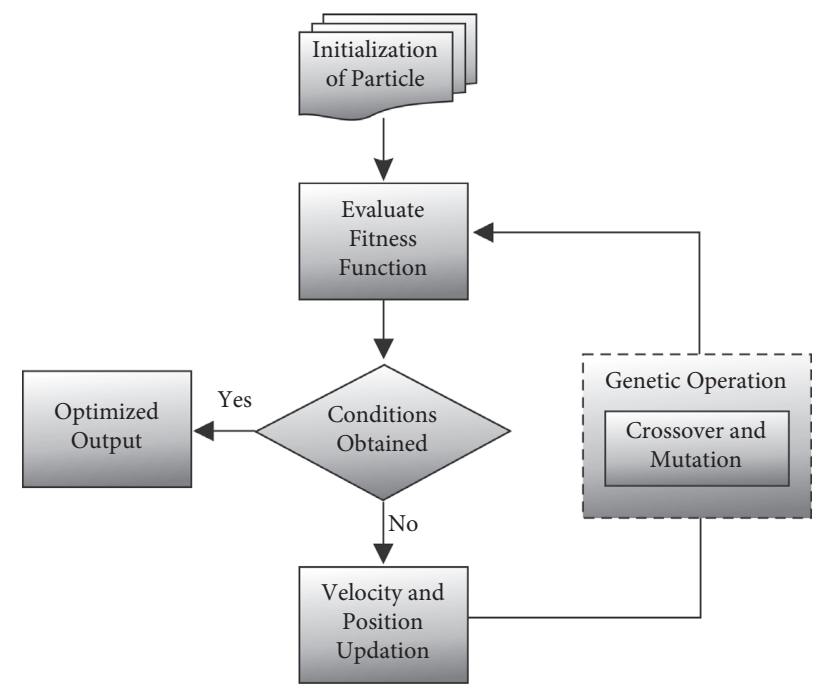

FIgURE 3: Flow diagram for HPSO with GA.

current particles array index and set the value as the new.

(2) Select the particle encompassing the best fitness value, and re-initialize its position. Together with this, assess the particle-containing the worst fitness value. And find whether its new place is acceptable or not. If it is in a good range, its position is updated, or a new position is allotted to the particle arbitrarily in its neighborhood. Then, refurbish the situation together with the velocity of other particles utilizing the following expression:

$$
\begin{aligned}
& x_{i}\left(t_{n}+1\right)=w * x_{i}\left(t_{n}\right)+c_{1} *\left[h_{i}^{*}(t)-h_{i}(t)\right]+c_{2} *\left[h_{g}^{*}(t)-h_{i}(t)\right], \\
& h_{i}\left(t_{n}+1\right)=h_{i}\left(t_{n}\right)+x_{i}\left(t_{n}+1\right),
\end{aligned}
$$

where $h_{i}^{*}(t)$ implies the best position of each particle, which signifies the private best objective (fitness) value hitherto obtained, and $h_{g}^{*}(t)$ implies the global best particle that signifies the best position among all particles on the populace, and $w$ signifies the inertia weight, that is used to maintain the particle; $h_{i}(t)$ denotes the position on the search space, $x_{i}\left(t_{n}\right)$ indicates the current velocity, and $c_{1}$ and $c_{2}$ are random numbers between $[0,1]$. The parameters $w, c_{1}$, and $c_{2}$ were considered $0.5,1.5$, and 1.5 , respectively.

(1) Subsequent to the calculation of velocity along with position, the crossover and mutation operations are performed to make the optimization more effective.

(2) Among different sorts of crossovers, the 2-point crossover is selected. In the 2-point crossover, 2 points are chosen upon the parent chromosomes utilizing Equations (10) and (11). The genes in between the 2 points are interchanged between the parent chromosomes. Consequently, children's chromosomes are gained. The crossover points are determined as

$$
\begin{aligned}
& x_{1}=\frac{\left|h_{i}(t)\right|}{3}, \\
& x_{2}=x_{1}+\frac{\left|h_{i}(t)\right|}{2} .
\end{aligned}
$$

(1) Now, the children's chromosomes are stocked up individually and also their corresponding indices are stored.

(2) Then, the mutation is accomplished by replacing several genes from every chromosome with new genes. The swapped genes are the randomly generated genes free of any recurrence inside the chromosome. The chromosomes chosen for crossover operation and the chromosomes obtained as 
a result of the mutation are then united, and the populace pool is filled with the chromosomes. attained as of the mutation are joined, and consequently, the populace pool is packed with the chromosomes.

The process is re-done until the solution with better fitness value is obtained.

Updating the particle's best position can well be specified as per the following equation:

$$
h_{i}\left(t_{n}+1\right)= \begin{cases}h_{i}^{*}\left(t_{n}\right), & S\left(h_{i}\left(t_{n}+1\right)\right) \geq S\left(h_{i}^{*}\left(t_{n}\right)\right), \\ h_{i}\left(t_{n}+1\right), & S\left(h_{i}\left(t_{n}+1\right)\right)<S\left(h_{i}^{*}\left(t_{n}\right)\right),\end{cases}
$$

The particle velocity at every dimension is limited to the interval $\left[ \pm X_{\max }\right.$, which is then measured and is contrasted with the $X_{\max }$. The $X_{\max }$ is a significant parameter. $X_{\max }$ helps in ascertaining the resolution with which the region between the present position and the target position is searched. Counting on the $X_{\max }$ values, the particles are pondered to offer the finest solution. The predefined equations are employed to gauge the fitness of the solution and to choose the better solution centered on these FV. The pseudocode for HPSO is presented in Figure 4.

After completing the optimization process, calculate the production function. Production function description is given next.

3.4. Production Function. Here, the aim is not merely to augment metabolic rate but to keep a large sum of alcohol within the process, and the economic cost could well be down. Additionally, other material risks can be reduced, ultimately being more convenient. Therefore, the following equation states the objective function, written as equation (4):

$$
y=C_{T G}(f), C_{D G}(f), C_{M G}(f), \frac{1}{C_{E}(f)}, \frac{1}{C_{A}(f)}, C_{G L} .
$$

Equation (13) shows the all-inclusive optimization function. This cost function equation elucidates that all products are minimized, excluding Ester and alcohol concentration.

\section{Results and Discussion}

The proposed optimized production of biodiesel is executed in the working platform of MATLAB (version 14) with machine configuration as follows: Intel Core i7 processor, 3.20 GHz CPU speed, and 4 GB RAM.

4.1. Comparison Analysis. The proposed method's performance is compared with respect to the concentration, fitness function, production function, and optimal temperature function that are conferred in this section.

Table 1 demonstrates the proposed HPSO performance with the existent PSO and GA centered on the production function. From Table 1, it can be noted that the HPSO has offered 0.2410 for 100 iterations, but the existing PSO

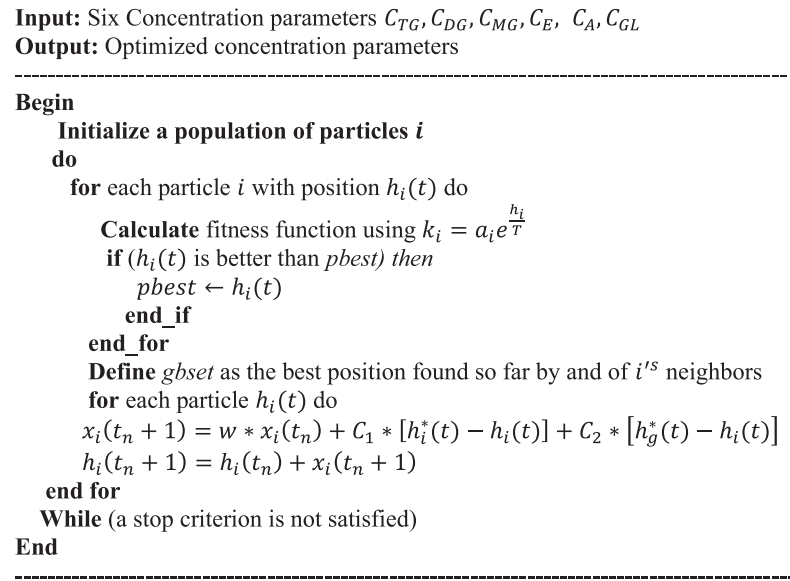

FIgURe 4: Pseudocode for hybrid PSO.

together with GA offer 0.0729 and 0.0351. Similarly, for 200 iterations, the proposed work gave 0.0591, whereas the current system provides 0.0071 for PSO and 0.0236 for GA. For 300 iterations, HSPO offers 0.1270 , but the existing optimization techniques have 0.0033 for PSO and 0.0155 for GA. Then, for 400 iterations, the proposed HPSO gave 0.3074 , but the current system provides 0.0729 for PSO and 0.0037 for GA. Finally, for 500 iterations, the proposed scheme offers 0.4030 , but the existing systems provide 0.0693 for the PSO optimization technique and 0.2970 for the GA optimization technique. Henceforth, from the predefined discussion, it can well be perceived that the HPSO-based optimized production of biodiesel is better when weighed down with the existent PSO together with GA. The graphical illustration results obtained are given in Figure 5. Table 2 shows the compared values production function of the proposed HPSO with existing PSO and GA based on production function.

Table 3 demonstrates the HPSO performance with the existing PSO and GA centered on the optimal temperature. The HPSO provides a temperature of $349.846 \mathrm{~K}$, but the current system provides $200.547 \mathrm{~K}$ for PSO and $273.674 \mathrm{~K}$ for the GA algorithm. Hence from this discussion, it is apparent that the HPSO technique is better than the existent techniques. The pictorial demonstration is exhibited in Figure 6.

Figure 7 illustrates the concentration versus iterations' performance, which shows that the concentration of the alcohol and the diglycerides is deficient, corresponding to the iterations. Similarly, all other four constituents increase in a stepwise manner. This shows that there was concentration minimization for two constituents and maximization for the other four constituents for better biodiesel production. Figure 8 illustrates the fitness with iterations' performance. The fitness function of the proposed algorithm $\left(f_{1}\right)$ was better than the existing GA $\left(f_{2}\right)$ and PSO $\left(f_{3}\right)$ in which the $f_{3}$ nearly attained the optimum fitness value after 100 iterations. Table 4 presents the production function value for 100 iterations for the optimized parameters $C_{T G}, C_{D G}, C_{M G}$, $C_{E}, C_{A}$, and $C_{G L}$ for HPSO, PSO, and GA. 
TABle 1: Coefficient of equation in Ref. [23].

\begin{tabular}{lccccc}
\hline$a_{6}$ & $a_{5}$ & $a_{4}$ & $a_{3}$ & $a_{2}$ & $a_{1}$ \\
$2.15 \mathrm{e} 4$ & $5.35 \mathrm{e} 3$ & $9.88 \mathrm{e} 10$ & $5.88 \mathrm{e} 12$ & $5.7 \mathrm{e} 5$ & $3.92 \mathrm{e} 7$ \\
$b_{6}$ & $b_{5}$ & $b_{4}$ & $b_{3}$ & $b_{2}$ & $b_{1}$ \\
4824.87 & 3231.18 & 7366.64 & 9993.96 & 4997.98 & 6614.83 \\
\hline
\end{tabular}

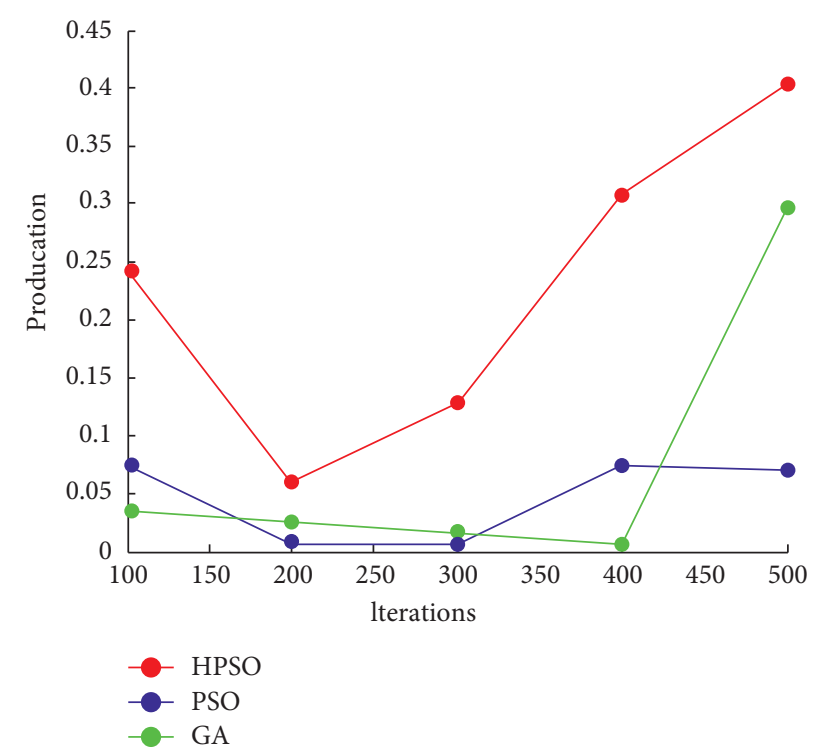

FIgURE 5: Production function of the proposed HPSO with existing PSO and GA based on production function.

TABLE 2: Comparison of the production function of the proposed HPSO with existing PSO and GA based on production function.

\begin{tabular}{lccc}
\hline Iterations & Proposed HPSO & Existing PSO & Existing GA \\
\hline 100 & 0.2410 & 0.0729 & 0.0351 \\
200 & 0.0591 & 0.0071 & 0.0236 \\
300 & 0.1270 & 0.0033 & 0.0155 \\
400 & 0.3074 & 0.0729 & 0.0037 \\
500 & 0.4030 & 0.0693 & 0.2970 \\
\hline
\end{tabular}

TABLE 3: The performance of the proposed HPSO with the existing PSO and GA based on optimal temperature.

\begin{tabular}{lr}
\hline Algorithm & Temperature \\
\hline Proposed HPSO & 349.846 \\
Existing PSO & 200.547 \\
Existing GA & 273.674 \\
\hline
\end{tabular}

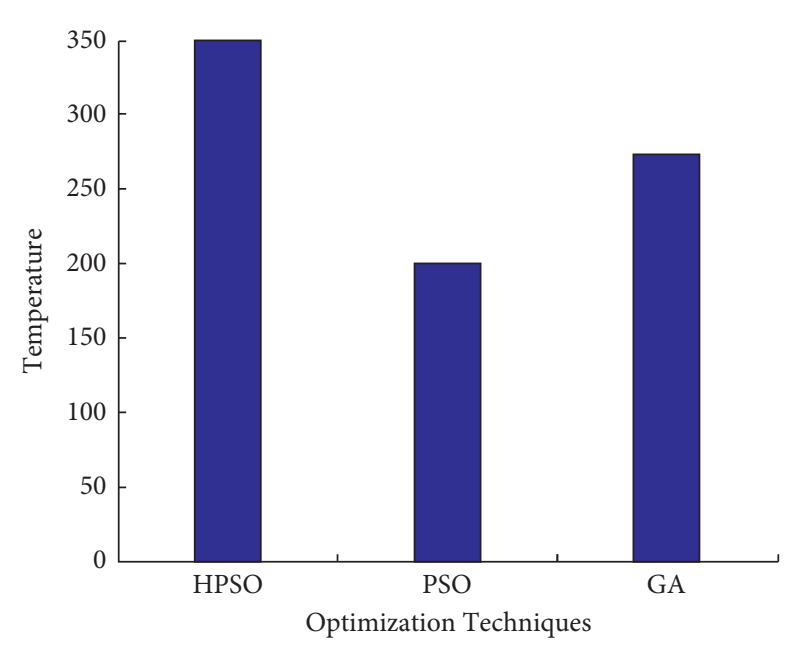

FIgURe 6: Performance of the proposed HPSO with existing PSO and GA based on optimal temperature. 


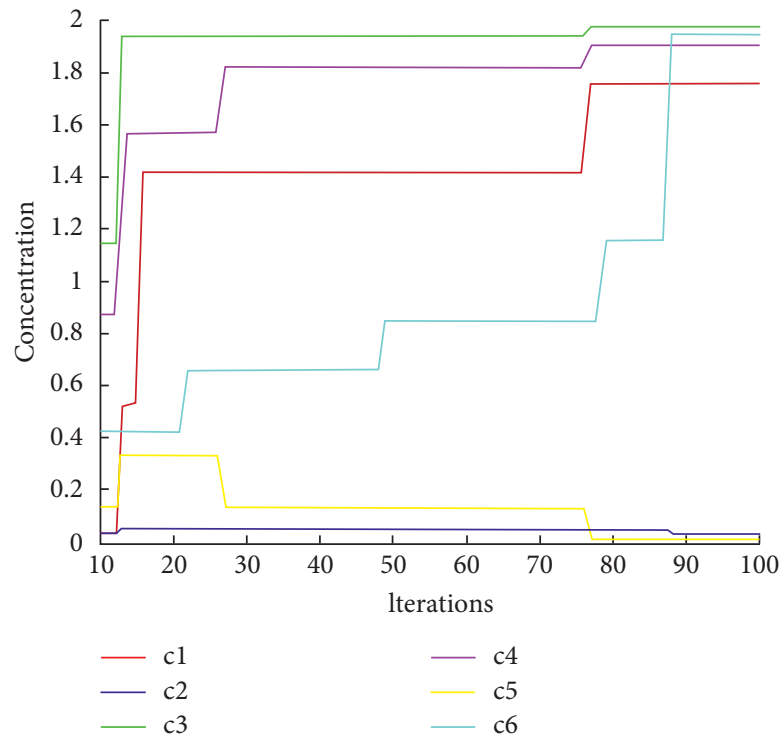

Figure 7: Performances of concentrations vs iterations.

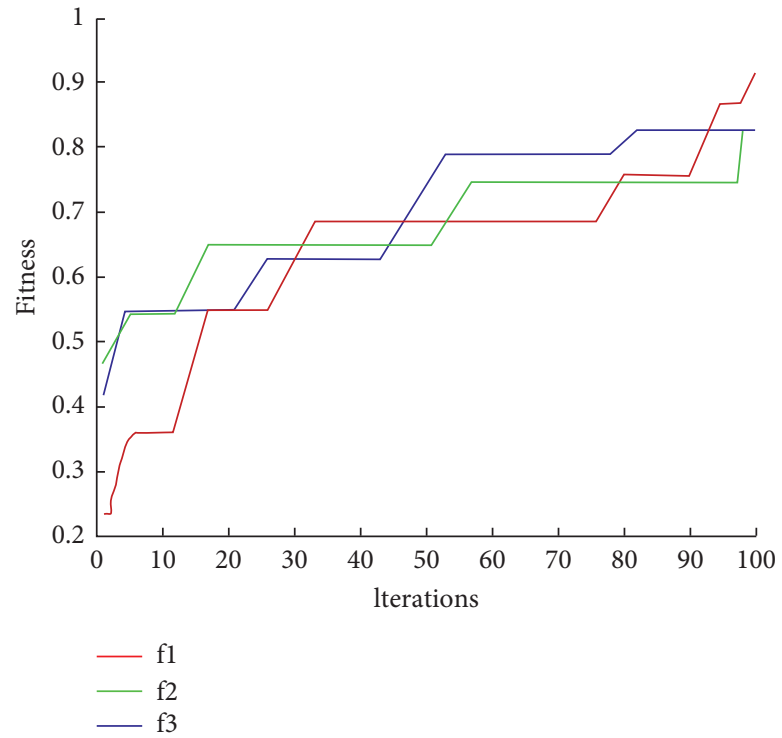

Figure 8: Performances of fitness vs iterations.

TABle 4: Production function value for 100 iterations.

\begin{tabular}{lccc}
\hline Optimized parameters & HPSO & PSO & GA \\
\hline$C_{T G}$ & 0.053745 & 0.086690 & 0.11320 \\
$C_{D G}$ & 0.068832 & 0.089045 & 0.119701 \\
$C_{M G}$ & 0.004334 & 0.003220 & 0.003850 \\
$C_{E}$ & 0.6642 & 0.52601 & 0.38385 \\
$C_{A}$ & 1.2709 & 1.40909 & 1.5501 \\
$C_{G L}$ & 5.110471 & 6.96235 & 1.64583 \\
Production function value & 0.2410 & 0.07305 & 0.0349 \\
\hline
\end{tabular}




\section{Conclusion}

The HPSO algorithm presented in this study is a practical approach to address biodiesel production, based on the above two calculation results of examples. It has excellent search capabilities and can produce a superior process route plan for the minimum completion time goal function. The following are the reasons: first, this algorithm established a coding approach that used direct coding based on product operations, which is more by the features of the problem for biodiesel production. It provides more detailed process information than the classic integrated and hierarchical coding methods, reduces the difficulty of decoding in, and improves the algorithm's efficiency and search performance. Second, the numerous genetic operation operators proposed in this article are better suited to biodiesel production.

A model for the optimized production of biodiesel using the HPSO algorithm is proposed. The PSO is hybridized with GA. To augment the fuel production during chemical reactions, an interaction between several substances has to be considered. Therefore, optimizing the reaction parameters can considerably increase productivity. The most critical parameters are the reaction temperature, which was monitored and optimized using HPSO. Here, the temperature value is arbitrarily generated from the IoT device.

Furthermore, the cost function is optimized utilizing the proposed algorithm. Thus, the proposed optimization algorithm contrasts with the existent PSO and GA concerning IOT sensed temperature and production function. For instance, the performance parameters, concentration, and fitness value are varied centric upon the number of iterations. Hence, the proposed optimized biodiesel production using the HPSO technique has effectively produced more biodiesel than other approaches.

\section{Data Availability}

The raw data supporting the conclusions of this article will be made available by the corresponding author without undue reservation.

\section{Conflicts of Interest}

The authors declare that they have no conflicts of interest.

\section{Authors' Contributions}

All authors listed have made a substantial, direct, and intellectual contribution to the work and approved it for publication.

\section{References}

[1] M. Valizadeh, S. Syafiie, and I. S. Ahamad, "Multi-objective particle swarm optimization for optimal planning of biodiesel supply chain in Malaysia," Advances in Intelligent Systems and Computing, vol. 287, pp. 293-302, 2014.

[2] C.-Y. Yu, L.-Y. Huang, I.-C. Kuan, and S.-L. Lee, “Optimized production of biodiesel from waste cooking oil by lipase immobilized on magnetic nanoparticles," International
Journal of Molecular Sciences, vol. 14, no. 12, pp. 24074-24086, 2013.

[3] H. Sanli, M. Canakci, and E. Alptekin, "Characterization of waste frying oils obtained from different facilities," in Proceedings of the World Renewable Energy Congress-Sweden, pp. 479-485, No. 057, Linköping; Sweden, May 2011.

[4] A. Gashaw and A. Teshita, "Production of biodiesel from waste cooking oil and factors affecting its formation: a review," International Journal of Renewable and Sustainable Energy, vol. 3, no. 5, pp. 92-98, 2014.

[5] M. A. Hanif, S. Nisar, M. N. Akhtar, N. Nisar, and N. Rashid, "Optimized production and advanced assessment of biodiesel: a review," International Journal of Energy Research, vol. 42, no. 6, pp. 2070-2083, 2018.

[6] T. Muppaneni, H. K. Reddy, S. Ponnusamy et al., "Optimization of biodiesel production from palm oil under supercritical ethanol conditions using hexane as co-solvent: a response surface methodology approach," Fuel, vol. 107, pp. 633-640, 2013.

[7] J. Yang, T. Astatkie, and Q. S. He, “A comparative study on the effect of unsaturation degree of camelina and canola oils on the optimization of bio-diesel production," Energy Reports, vol. 2, pp. 211-217, 2016.

[8] A. E. Atabani, A. S. Silitonga, I. A. Badruddin, T. M. I. Mahlia, H. H. Masjuki, and S. Mekhilef, "A comprehensive review on biodiesel as an alternative energy resource and its characteristics," Renewable and Sustainable Energy Reviews, vol. 16, no. 4, pp. 2070-2093, 2012.

[9] D. Righini, F. Zanetti, and A. Monti, “The bio-based economy can serve as the springboard for camelina and crambe to quit the limbo," OCL, vol. 23, no. 5, Article ID D504, 2016.

[10] I. A. Musa, "The effects of alcohol to oil molar ratios and the type of alcohol on biodiesel production using transesterification process," Egyptian Journal of Petroleum, vol. 25, no. 1, pp. 21-31, 2016.

[11] D. O. Onukwuli, L. N. Emembolu, C. N. Ude, S. O. Aliozo, and M. C. Menkiti, "Optimization of biodiesel production from refined cotton seed oil and its characterization," Egyptian Journal of Petroleum, vol. 26, no. 1, pp. 103-110, 2017.

[12] K. Okitsu, Y. Sadanaga, N. Takenaka, Y. Maeda, and H. Bandow, "A new co-solvent method for the green production of biodiesel fuel-optimization and practical application," Fuel, vol. 103, pp. 742-748, 2013.

[13] D. Ghayal, A. B. Pandit, and V. K. Rathod, "Optimization of biodiesel production in a hydrodynamic cavitation reactor using used frying oil," Ultrasonics Sonochemistry, vol. 20, no. 1, pp. 322-328, 2013.

[14] L. A. W. da Silva and L. dos Santos Coelho, "An adaptive particle swarm approach applied to optimization of a simplified supply chain," in 19th International Conference on Production Research, 2007.

[15] H. Xiong, X. Guo, and W. Xie, "Biodiesel remote monitoring system design based on IOT," in Proceedings of the International Conference on Human Centered Computing, pp. 750-756, Phnom Penh, Cambodia, 2014, November.

[16] G. Antony Miraculas, N. Bose, and R. Edwin Raj, "Process parameter optimization for biodiesel production from mixed feedstock using empirical model," Sustainable Energy Technologies and Assessments, vol. 28, pp. 54-59, 2018.

[17] P. Adewale, L. N. Vithanage, and L. Christopher, "Optimization of enzyme-catalyzed biodiesel production from crude tall oil using Taguchi method," Energy Conversion and Management, vol. 154, pp. 81-91, 2017. 
[18] M. Saqib, M. W. Mumtaz, A. Mahmood, and M. I. Abdullah, "Optimized biodiesel production and environmental assessment of produced biodiesel," Biotechnology and Bioprocess Engineering, vol. 17, no. 3, pp. 617-623, 2012.

[19] S. Dhingra, K. K. Dubey, and G. Bhushan, "A polymath approach for the prediction of optimized transesterification process variables of polanga biodiesel," Journal of the American Oil Chemists' Society, vol. 91, no. 4, pp. 641-653, 2014.

[20] S. H. Yeom and Y. W. Go, "Optimization of a novel two-step process comprising re-esterification and transesterification in a single reactor for biodiesel production using waste cooking oil," Biotechnology and Bioprocess Engineering, vol. 23, no. 4, pp. 432-441, 2018.

[21] M. Mubarak, A. Shaija, and T. V. Suchithra, "Optimization of lipid extraction from Salvinia molesta for biodiesel production using RSM and its FAME analysis," Environmental Science and Pollution Research, vol. 23, no. 14, pp. 14047-14055, 2016.

[22] Z. Wen, S. Hu, D. De Clercq et al., "Design, implementation, and evaluation of an Internet of Things (IoT) network system for restaurant food waste management," Waste Management, vol. 73, pp. 26-38, 2018.

[23] P. T. Benavides and U. Diwekar, "Optimal control of biodiesel production in a batch reactor," Fuel, vol. 94, pp. 211-217, 2012.

[24] V. Ramasamy and S. Thalavai Pillai, "An effective HPSOMGA optimization algorithm for dynamic resource allocation in cloud environment," Cluster Computing, vol. 23, no. 3, pp. 1711-1724, 2020. 